\title{
Cool carbon stars in the halo: new very red or distant objects ${ }^{\star} \star \star$
}

\author{
N. Mauron ${ }^{1}$, K. S. Gigoyan ${ }^{2}$, and T. R. Kendall ${ }^{3}$ \\ 1 GRAAL, CNRS and Université Montpellier II, Place Bataillon, 34095 Montpellier, France \\ e-mail: mauron@graal .univ-montp2.fr \\ 2378433 Byurakan Astrophysical Observatory and Isaac Newton Institute of Chile, Armenian Branch, Ashtarak d-ct, Armenia \\ e-mail: kgigoyan@bao.sci.am \\ 3 Centre for Astrophysics Research, Science and Technology Research Institute, University of Hertfordshire, College Lane, \\ Hatfield AL10 9AB, UK \\ e-mail: trkendall@googlemail.com
}

Received 28 June 2007 / Accepted 23 August 2007

\begin{abstract}
Aims. The goal of this paper is to present and analyse a new sample of cool carbon (C) stars located in the Galactic halo. Methods. These rare objects are discovered by searching the 2MASS point-source catalogue for candidates having near-infrared colours typical of $\mathrm{C}$ stars. Optical spectroscopy is subsequently performed.

Results. Twenty-three new $\mathrm{C}$ stars were discovered, with their $K_{\mathrm{s}}$ magnitude in the range 6 to 13.3. Spectra are typical of N-type carbon stars with $\mathrm{C}_{2}$ and $\mathrm{CN}$ bands and sometimes $\mathrm{H} \alpha$ in emission. One object is a S-type star. When the objects are bright enough $(V \leq 15.5)$, the data of the Northern Sky Variability Survey can be exploited. In all cases, stars belonging to this survey show light variations confirming that they are AGB stars. Distances and galactocentric $X Y Z$ coordinates have been estimated by assuming these stars to be similar in luminosity to those of the Sagittarius dwarf galaxy. Four objects are particularly red with $J-K_{\mathrm{s}}>3$, with two located at more than $5 \mathrm{kpc}$ from the Galactic plane. Eight additional objects with similar properties are found in the literature and our previous works. These $12 \mathrm{C}$ stars could be useful to study mass loss at low metallicity. Two other objects are remarkably far from the Sun, at distances of 95 and $110 \mathrm{kpc}$. They are located, together with two other C stars previously found, in the region $Z<-60 \mathrm{kpc}$ in which the model of Law et al. (2005) predicts the Sgr Stream to have a loop.
\end{abstract}

Key words. stars: carbon - Galaxy: halo - Galaxy: stellar content

\section{Introduction}

Cool carbon (C) stars are objects evolving on the asymptotic giant branch (AGB). This is a brief phase of stellar evolution characterized by high luminosity, low effective temperature, and the appearance at the surface of the star of nuclear products fabricated in its internal layers (for reviews on AGB and C stars, see Habing 1996; Wallerstein \& Knapp 1998). The progenitors of these $\mathrm{C}$ stars have intermediate masses, from about $1.3 M_{\odot}$ in most cases, up to about $4 M_{\odot}$. C stars are variable sources. They can be recognized by their very red colours and the strong spectral features arising from carbon-rich molecules. Because evolution on the AGB is fast, they are not very abundant in the Galactic disc. However, because of their luminosity, they have been detected and studied in many galaxies of the Local Group and beyond. They are more numerous in systems with an average low metallicity, and generally indicate the presence of an intermediate age population (see for example Wallerstein \& Knapp 1998; Van den Bergh 2000; Mouhcine \& Lançon 2001; Groenewegen 2005).

In the Galactic disc and close to the Sun, i.e. at distances less than $\sim 5 \mathrm{kpc}$, AGB C stars have a scale height of $200 \mathrm{pc}$ (Claussen et al. 1987; Groenewegen et al. 1992; Bergeat et al. 2002).

\footnotetext{
^ Based on observations done at Haute Provence Observatory operated by the Centre National de Recherche Scientifique (France), and at Byurakan Observatory (Armenia).

$\star \star$ Appendix A is only available in electronic form at http://www. aanda.org
}

Therefore, very few of them are expected at more than about $\sim 1 \mathrm{kpc}$ above or below the Galactic plane. In the old halo population, all stars with masses similar to those of $\mathrm{C}$ stars have evolved beyond the luminous AGB phase. However, it has long been known that some faint and cool carbon stars do exist at large distances and at high galactic latitude. Totten and Irwin (1998, hereafter TI98) listed 41 previously known objects, this number including some warmer CH-type objects. They discovered 36 additional cases, with $29 \mathrm{~N}$-type and 7 CH-type (for more information about classification of $\mathrm{C}$ stars, see Wallerstein \& Knapp 1998). One of their goals was to show that these halo $C$ stars might arise from the disruption of dwarf galaxies captured by the Galaxy. This was eventually proved by Ibata et al. (2001) who found that more than half of these $\mathrm{C}$ stars are coherently clustered on a great circle on the sky, and trace the debris stream of the Sagittarius dwarf galaxy.

The goal of this paper is to present new discoveries of halo cool C stars and to study their properties. In our previous works (Mauron et al. 2004, 2005), we followed the approach of TI98 to search for these rare halo cool C stars. However, rather than using visible photometry from photographic plates to select candidates, we have exploited the recent 2MASS near-infrared survey and its point-source catalogue (Cutri et al. 2003). This survey provides us with accurate $J H K_{\mathrm{s}}$ photometry, enabling the potential discovery of $\mathrm{C}$ stars via their near-infrared colours. Followup spectroscopy is then performed to determine whether these candidates are carbon-rich, and show the expected signatures of evolved AGB objects. 
A first result of our research was that 50 new cool halo $\mathrm{C}$ stars were discovered. This suggested that, firstly, the observational strategy was correct (for details on the candidate selection process, see Mauron et al. 2005). Secondly, it was found that a majority of these objects belong to the Sagittarius (Sgr) stream, but not all of them do, in agreement with previous findings. Finally, we found several remarkably faint $\left(K_{\mathrm{s}} \geq 11\right) \mathrm{ob}-$ jects; interesting probes of the halo at distances from the Sun as large as $\sim 60-120 \mathrm{kpc}$.

In this paper, we report on our continuing program to enlarge the sample of these halo $\mathrm{C}$ stars. We wish eventually to establish a complete $K_{\mathrm{s}}$-band limited inventory. Two new very distant sources were discovered with $K_{\mathrm{s}}>12$. Observations and data reduction are described in Sect. 2. In Sect. 3 we analyse our new sample of 25 halo $\mathrm{C}$ stars, and we discuss global properties of the sample and individual cases, including consideration of colours, spectra and variability. Very red sources are discussed in Sect. 4. In Sect. 5, we estimate the distances of the sources and discuss their location in the Galactic halo. A summary of this work and our conclusions are finally given in Sect. 6.

\section{Observations}

Slit spectroscopy of our candidates was performed at the Byurakan Observatory (Armenia) and at Haute-Provence Observatory (France). At Byurakan, the observations were carried out at various dates during 2005. The instrument was the SCORPIO spectrograph attached to the $2.6-\mathrm{m}$ telescope. The spectrograph was used with a $600 \mathrm{~g} \mathrm{~mm}^{-1}$ grating and a Lick-3 $2064 \times 2058 \mathrm{CCD}$ detector which has $15 \times 15 \mu \mathrm{m}$ pixels. The resulting dispersion is $1.7 \AA$ per pixel. The resolving power is $\lambda / \delta \lambda=750$, hence $\delta \lambda=8 \AA$ at $6000 \AA$. The useful spectral region is from $4600 \AA$ to $7000 \AA$.

At Haute-Provence, the observations were made during the nights September 5 to 9, 2005 and September 27 to October 2, 2006. The instrument was the CARELEC spectrograph mounted at the Cassegrain focus of the 1.93-m telescope. The spectrograph was used with a $150 \mathrm{~g} \mathrm{~mm}^{-1}$ grating and an EEV $2048 \times 1024$ CCD chip with $13.5 \times 13.5 \mu$ m pixels. The dispersion is $3.6 \AA$ per pixel. The slit width was 2.0 arcsec. The resolving power is $\lambda / \delta \lambda=460$, and $\delta \lambda=13 \AA$ at $6000 \AA$, with a spectral coverage of 4400 to $8400 \AA$.

Typical exposure times were from about 1 minute for the brightest candidate carbon stars $(R \sim 12)$ to about $1 \mathrm{~h}$ for the faintest ones $(R \sim 17)$. The low spectral resolution was chosen to allow spectroscopy of these faint stars in a reasonable time, and the spectra have sufficient signal to noise ratio $(\geq 20)$ to perfectly recognize the strong molecular features of carbon-rich objects. Most of the contaminants in our list of $\mathrm{C}$ candidates are cool $\mathrm{M}$ giants or $\mathrm{M}$ dwarfs. Faint sources required clear conditions with good seeing. When these conditions were not fulfilled, only bright sources were observed.

Standard data reduction for slit spectroscopy was carried out with the ESO Midas software. It included bias subtraction, flatfielding, extraction of one-dimensional spectra for the object and for the sky, subtraction of the sky spectrum, cleaning of cosmic rays, and wavelength calibration. Then, a correction was applied to take into account the instrumental spectral efficiency, and this was done with the spectrum of a standard photometric star observed with the same instrumental set-up. The spectra are thus proportional to a flux expressed in erg s $\mathrm{s}^{-1} \mathrm{~cm}^{-2} \AA^{-1}$ and can be compared to those shown by TI98. However, no absolute calibration could be achieved because the sky was most of the time

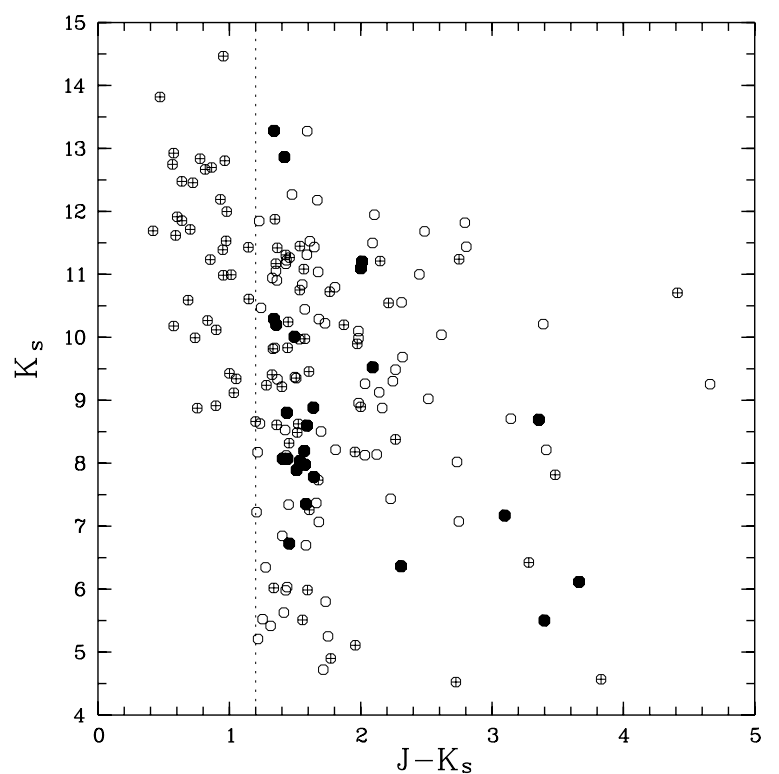

Fig. 1. Colour-magnitude diagram with $K_{\mathrm{s}}$ plotted versus $J-K_{\mathrm{s}}$ for known cool halo $\mathrm{C}$ stars. Filled circles are objects of this work. Encircled crosses are the objects listed in TI98. Empty circles are objects found in other previous studies, with most of them being from Mauron et al. $(2004,2005)$. The vertical dotted line at $J-K_{\mathrm{s}}=1.2$ approximatively separate the $\mathrm{CH}$-type stars to the left and the cool $\mathrm{N}$-type stars to the right.

not photometric, and because strong slit losses occured when the seeing was bad. Therefore, only relative spectral distributions can finally be considered and are drawn in our plots.

\section{Results}

\subsection{Properties of the sample}

Table 1 lists the main parameters of the 25 objects that are under analysis in this work. In the first column, the object serial number is given, following the numbering of Table 1 of Mauron et al. (2005). Coordinates $\alpha$ and $\delta$ (J2000) are given in the object 2MASS J names as HHMMSS.ss \pm DDMMSS.s. The quantities $l, b$ are galactic coordinates in degrees. The $B \& R$ magnitudes are from the USNO-A2.0 catalogue (Monet et al. 1998) as given in the 2MASS catalogue. If these data are not available, we have adopted the $B$ and $R$ values given in the APM database (Irwin 2000), or we have estimated them from the USNO-B1.0 data (Monet et al. 2003). Uncertainties on $B$ and $R$ of the order of $0.4 \mathrm{mag}(1 \sigma)$. The $J H K_{\mathrm{s}}$ magnitudes and the $J-K_{\mathrm{s}}$ colour are from the 2MASS catalogue, with uncertainties on $J H K_{\mathrm{s}}$ of about $0.02-0.03$ mag in most cases.

One can see in Table 1 that the $R$ magnitudes are in the range $\sim 11$ up to $\sim 17$. Nine sources are brighter than $R=13.0$ and three are fainter than 16.0. The $B-R$ colour is often greater than 3.0. More precisely, of the 25 objects, 20 have their $B-R$ greater than 3.0. Here, it is interesting to note that if $B-R$ had been requested to be larger than 3.0 in our selection process, we would have missed the two most interesting objects (\#59 and \#81), with the faintest $K_{\mathrm{s}}$ magnitude and the largest distances (see below).

The listed $K_{\mathrm{s}}$ magnitudes cover the range $\sim 6$ to $\sim 13$. All objects have $J-K_{\mathrm{s}}>1.30$. Figure 1 shows the colour-magnitude diagram of our sample. The objects of Table 1 are plotted with filled circles. The encircled crosses show the 77 objects listed in TI98, and the empty circles indicate objects found in previous 
Table 1. List of observed carbon stars and their main characteristics. The columns give the running number, the $2 \mathrm{MASS}$ name, the galactic coordinates, the $B$ and $R$ magnitudes and the $B-R$ colour index, the $J H K_{\mathrm{s}}$ magnitudes from 2MASS and the $J-K_{\mathrm{s}}$ colour index.

\begin{tabular}{|c|c|c|c|c|c|c|c|c|c|c|c|}
\hline No. & 2MASS name & $l$ & $b$ & $B$ & $R$ & $B-R$ & $J$ & $H$ & $K_{\mathrm{s}}$ & $J-K_{\mathrm{s}}$ & Note \\
\hline 59 & 2MASS J003504.77+010845.8 & 114.351 & -61.453 & 19.2 & 16.7 & 2.5 & 14.620 & 13.734 & 13.280 & 1.340 & \\
\hline 60 & 2MASS J011256.39+395945.3 & 127.393 & -22.689 & 15.1 & 11.3 & 3.8 & 8.181 & 7.160 & 6.725 & 1.456 & \\
\hline 61 & 2MASS J014736.29+371229.1 & 135.175 & -24.325 & 17.4 & 13.3 & 4.1 & 10.523 & 9.436 & 8.884 & 1.639 & \\
\hline 62 & 2MASS J020056.14+094535.6 & 149.845 & -49.446 & 19.7 & 15.2 & 4.5 & 10.266 & 8.525 & 7.170 & 3.096 & \\
\hline 63 & 2MASS J021012.06-015738.9 & 163.123 & -58.546 & 16.2 & 12.2 & 4.0 & 11.505 & 10.605 & 10.010 & 1.495 & 1 \\
\hline 64 & 2MASS J030011.33+164940.2 & 162.049 & -36.082 & 17.5 & 14.2 & 3.3 & 11.632 & 10.742 & 10.293 & 1.339 & \\
\hline 65 & 2MASS J034828.12+165703.2 & 172.347 & -28.475 & 18.1 & 14.8 & 3.3 & 13.098 & 11.903 & 11.097 & 2.001 & \\
\hline 66 & 2MASS J035010.66+260502.8 & 165.713 & -21.582 & 16.7 & 13.3 & 4.4 & 10.240 & 9.244 & 8.803 & 1.437 & \\
\hline 67 & 2MASS J040143.35+084210.6 & 181.911 & -31.637 & 16.1 & 12.6 & 3.5 & 9.765 & 8.703 & 8.195 & 1.570 & \\
\hline 68 & 2MASS J040648.84+162818.3 & 176.133 & -25.619 & 16.9 & 13.0 & 3.9 & 9.573 & 8.517 & 8.036 & 1.537 & \\
\hline 69 & 2MASS J042638.77+142516.1 & 181.245 & -23.296 & 19.2 & 17.1 & 2.1 & 13.215 & 12.030 & 11.204 & 2.011 & 2 \\
\hline 70 & 2MASS J045602.71+092219.1 & 190.204 & -20.452 & 17.3 & 13.4 & 3.9 & 10.189 & 9.141 & 8.599 & 1.590 & \\
\hline 71 & 2MASS J060634.52+731026.9 & 140.861 & +22.738 & 15.9 & 12.7 & 3.2 & 9.403 & 8.393 & 7.891 & 1.512 & \\
\hline 72 & 2MASS J064618.05+543133.6 & 161.381 & +21.086 & 14.8 & 12.8 & 2.0 & 9.478 & 8.458 & 8.072 & 1.406 & \\
\hline 73 & 2MASS J071057.47+475818.0 & 169.337 & +22.917 & 16.1 & 12.3 & 3.8 & 8.935 & 7.880 & 7.352 & 1.583 & \\
\hline 74 & 2MASS J171755.52+043606.9 & 26.142 & +22.867 & 17.3 & 14.5 & 2.8 & 11.557 & 10.592 & 10.200 & 1.357 & \\
\hline 75 & 2MASS J175815.64+223551.1 & 48.195 & +21.356 & 15.7 & 12.0 & 3.7 & 9.516 & 8.632 & 8.073 & 1.443 & \\
\hline 76 & 2MASS J193930.23+754140.5 & 107.672 & +23.332 & 16.2 & 12.5 & 3.7 & 9.426 & 8.326 & 7.783 & 1.643 & \\
\hline 77 & 2MASS J195840.16+774526.2 & 110.190 & +23.019 & 21.0 & 14.9 & 6.1 & 12.048 & 10.161 & 8.692 & 3.356 & \\
\hline 78 & 2MASS J201559.67+763508.4 & 109.469 & +21.659 & 16.9 & 12.9 & 4.0 & 9.553 & 8.573 & 7.978 & 1.575 & \\
\hline 79 & 2MASS J210522.23+780116.2 & 112.308 & +20.130 & 17.0 & 13.0 & 4.0 & 9.779 & 7.699 & 6.116 & 3.663 & \\
\hline 80 & 2MASS J211944.93+180029.7 & 68.200 & -21.673 & 18.1 & 13.6 & 4.5 & 8.671 & 7.338 & 6.364 & 2.307 & 3 \\
\hline 81 & 2MASS J212318.24-140819.5 & 37.019 & -39.965 & 18.7 & 16.3 & 2.4 & 14.281 & 13.427 & 12.862 & 1.419 & \\
\hline 82 & 2MASS J215526.97+234214.4 & 78.911 & -23.760 & 18.3 & 14.0 & 4.3 & 8.901 & 6.986 & 5.503 & 3.398 & \\
\hline 83 & 2MASS J222301.20+221656.5 & 83.298 & -28.940 & 20.2 & 15.0 & 5.2 & 11.685 & 10.392 & 9.524 & 2.161 & \\
\hline
\end{tabular}

Notes: (1) carbon star identified by TI98. It was reobserved because of its peculiar blue index $B-R=-0.6$ in USNOC-A2.0; (2) carbon star identified by Cruz et al. (2003). A first spectrum is shown in this work; (3) this object is a S-type evolved object.

works aimed at discovering new faint and cool halo $\mathrm{C}$ stars (e.g., Mauron et al. 2004, 2005; Liebert et al. 2000). The vertical line at $J-K_{\mathrm{s}}=1.20$ is the approximate limit between $\mathrm{CH}$-type stars at left and cool AGB C stars at right. Only cool N-type objects were searched in the present study. In this diagram, a cool C star located at a distance of $50 \mathrm{kpc}$ like the Large Magellanic Cloud (hereafter LMC) has a typical magnitude $K_{\mathrm{s}} \approx 10.8$. One can note in Fig. 1 that the number of objects in the upper right region $J-K_{\mathrm{s}}>1.2, K_{\mathrm{s}} \gtrsim 11$ has progressively increased, with in particular two objects, \#59 and \#83, which have $K_{\mathrm{s}}=13.28$ and $K_{\mathrm{s}}=12.86$ respectively.

There are two objects that were already known as carbon stars. One is \#63, that was already in TI98 under the name C*30 or APM 0207-0211. It was reexamined by us because of its intriguing colour $B-R=-0.6$ in the USNO-A2.0 catalog. Usually, this colour for cool $\mathrm{C}$ stars covers the range from 2.5 to $\sim 4$, or even redder. We wished to detect a possible blue excess near $4500 \AA$, but found none. Our spectrum is very similar to that of TI98, the only difference being the presence of a small $\mathrm{H} \alpha$ emission at $6563 \AA$. Because the APM catalogue gives $R=12.16$ and $B_{J}-R=4.08$ (adopted in Table 1 ), our conclusion is that the USNO-A2.0 data is probably simply flawed.

The other object which was known as a $\mathrm{C}$ star is \#69. This object was found by Cruz et al. (2003) during their search for brown dwarfs. It was observed by us to obtain a first spectrum and because it was faint, $K_{\mathrm{s}}=11.2$, implying a large distance.

\subsection{Spectra}

All obtained spectra are presented in the on-line Appendix. In Fig. 2, we show only the spectrum of the most distant star (\#59).

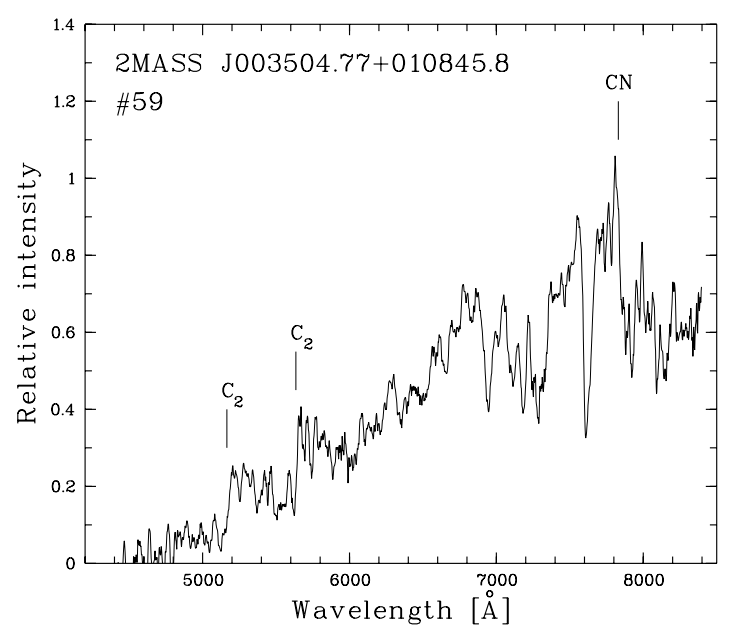

Fig. 2. Spectrum of object \#59, the faintest source in the $K_{\mathrm{s}}$ band $\left(K_{\mathrm{s}}=13.28, R=16.7\right)$. The spectrum is typical of cool N-type stars, with a flux increasing with wavelength, and with characteristic molecular features, i.e. $\mathrm{C}_{2}$ bands at $\lambda<6000 \AA$ and the flux break at $\lambda \approx 7800 \AA$ due to $\mathrm{CN}$.

This spectrum has the lowest $\mathrm{S} / \mathrm{N}$ in our sample, but the characteristics of a cool $\mathrm{C}$ star, such as a rising continuum and the $\mathrm{C}_{2}$ and $\mathrm{CN}$ bands, are obvious. One object, \#80, is a S-type star, with strong VO absorption near $7400 \AA$. Its H $\alpha$ (6563 $\mathrm{A}$ ) line is in emission and this star displays important light variations (see below). Of the $25 \mathrm{C}$ stars observed, 10 show the $\mathrm{H} \alpha$ line in emission, representing $40 \%$ of the sample, which is in agreement with previous findings of Mauron et al. (2004, 2005). 

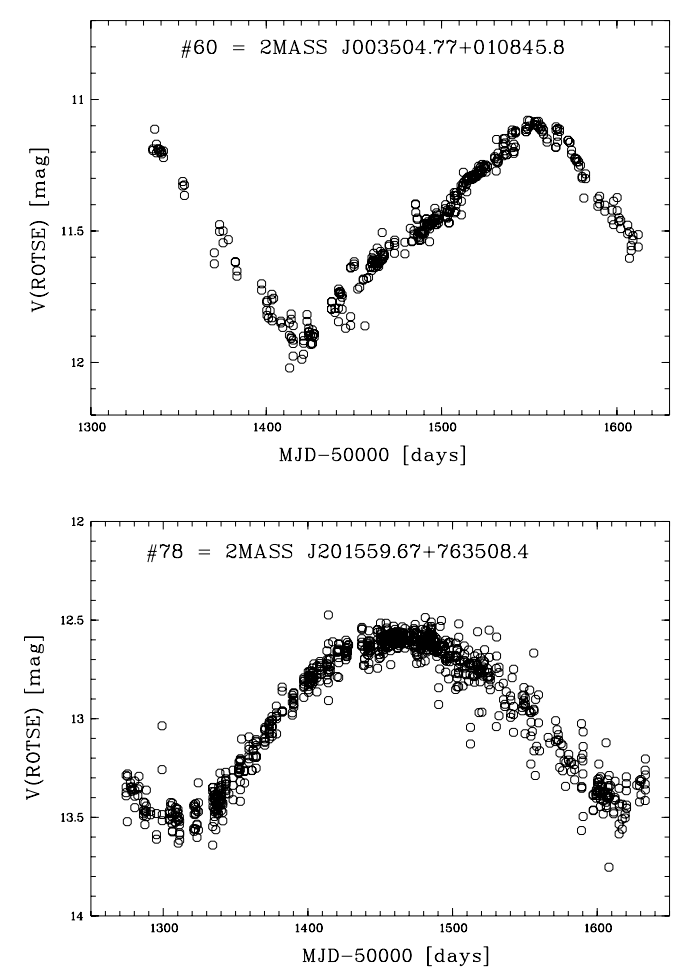

Fig. 3. Light curves for objects \#60 and \#78, from NSVS data. Only the general trend should be considered and a few outliers have to be ignored.

\subsection{Variability}

In order to study variability of our objects, we have considered the Northern Sky Variability Survey (NSVS) of Woźniak et al. (2004). This survey provides light curves for sources with $V$ band magnitudes of 8 to $\sim 15.5$. The length of each record is typically 300 days. Some data points are obvious outliers and should be ignored. Only the general trend of the light curves must be considered. Not all the objects in our sample have a counterpart in the NSVS, especially the faintest ones. In all cases in which data is available and sufficiently numerous, they suggest regular or irregular variations with $V$-band amplitudes of about 1 mag. up to several magnitudes. For illustration, Fig. 3 shows the light curves of objects \#60 and \#78. Light curves for objects \# 66, 71, $73,75,76,78,79,80,82$, and 83 are given in the on-line appendix. Note that the $\mathrm{S}$ star (\# 80) displays the largest variation and linearly decreases from $V=12.5$ to $V=15.0$ over a period of 200 days.

\section{Very red carbon stars in the halo}

One of the result of this paper is the discovery of four very red $\mathrm{C}$ stars seen in Fig. 1 as filled circles with $J-K_{\mathrm{s}}$ between 3 and 4. By searching the literature about high latitude, cool and faint C stars, we have found 8 other objects out of the Galactic plane having a similarly red $J-K_{\mathrm{s}}$ colour, $K_{\mathrm{s}} \gtrsim 5.5$, and $|b|>20^{\circ}$. In our research for these stars, we also require the IRAS $12 \mu \mathrm{m}$ flux to be less than $\sim 7 \mathrm{Jy}$ in order to exclude nearby, very dusty objects like IRC +10216 or AFGL 3068. The data for this sample of 12 cases are listed in Table 2. Object \#82 has no $12 \mu \mathrm{m}$ flux because IRAS scans do not pass over a narrow lane in which this source lies. For 2MASS J194850.65-305831.7, the $12 \mu \mathrm{m}$ data is a conservative upper limit derived from the examination of the
IRAS Faint Source catalogue sources close to it. Distances to the Sun and from the Galactic plane were derived as explained below. One can see that the stars \#79 and \#82 are moderatly out of the plane by $1.3 \mathrm{kpc}$, but all others are clearly at very large heights, and this remains true even if our distances are overestimated by $\sim 40 \%$ ( $-2 \sigma$, see next section).

The best studied case is IRAS $12560+1656$, discovered by Beichman et al. (1990) in a sample of faint IRAS sources located at high $b$ and having faint counterparts in the POSS plates. This object has been further investigated by Groenewegen et al. (1997) who found a remarkably low expansion velocity of its circumstellar envelope, measured in $\mathrm{CO}\left(3 \mathrm{~km} \mathrm{~s}^{-1}\right)$. They also found that this object is deficient in oxygen relative to the Sun, by $\sim 0.7$ dex. IRAS $08546+1732$, not detected in CO, is deficient as well. Therefore, our 12 objects deserve interest because it is established that already two of them are metal-poor, with one having very unusual wind properties. Because these 12 objects do not belong to the Galactic disc, they may come from the debris of the Sgr dwarf and have a metal abundance $[\mathrm{Fe} / \mathrm{H}]$ around -1.0 (Van den Bergh 2000).

Are their infrared colours different from those of C stars having a much higher $12 \mu \mathrm{m}$ flux? In order to test that, we considered the sample of 330 bright infrared C stars of Groenewegen et al. (2002). Most of these stars are close to the Galactic plane and might suffer some interstellar extinction in $J$ and $K_{\mathrm{s}}$. So we selected a subsample of their stars with $|b|>10^{\circ}$. Their $f_{12}$ fluxes are between 13 and 250 Jy. Figure 4 shows the colour-colour plot with $K_{\mathrm{s}}-$ [12] versus $J-K_{\mathrm{s}}$. The [12] magnitude is taken to be zero for Vega, thus [12] $=-2.5 \log _{10}\left(f_{12} / 41.6\right)$, with $f_{12}$ in Jy. This plot shows that the objects in Table 2 are located on the sequence of ordinary disc $\mathrm{C}$ stars going from warm cases at lower left to very cool dusty objects at upper right. The 12 objects of Table 2 do not seem different from brighter objects as far as the $J-K_{\mathrm{s}}$ and $K_{\mathrm{s}}$-[12] colours are concerned.

To conclude, in addition to IRAS $12560+1656$ and IRAS $08546+1732$ quoted above, the 10 other stars of Table 2 may be useful to study mass loss of low metallicity AGB C stars. They are generally much closer to the Sun than similar carbon stars in the Magellanic Clouds.

\section{Distances and location in the halo}

The distances of our objects were estimated by using their observed $J-K_{\mathrm{s}}$ colour and $K_{\mathrm{s}}$ magnitude. The $K_{\mathrm{s}}$ band is used because it is less sensitive to temporal variations than other filters and suffers less interstellar extinction. Absolute magnitudes were determined as in Mauron et al. (2004, 2005). Briefly, $M_{\mathrm{K}_{\mathrm{s}}}$ values are obtained by considering the $K_{\mathrm{s}}$ magnitudes of LMC carbon stars averaged over colour bins of $0.1 \mathrm{mag}$ and adding $0.5 \mathrm{mag}$. The colour-magnitude diagram $K_{\mathrm{s}}$ versus $J-K_{\mathrm{s}}$ of the LMC can be seen in Fig. 3 of Nikolaev and Weinberg (2000). The supplementary 0.5 -mag term is due to the average difference between C stars in the LMC and C stars in the Sgr dwarf galaxy (see Mauron et al. 2004, for details). This calibration on the $\mathrm{C}$ stars of Sgr is adopted because a majority of halo $\mathrm{C}$ stars originate from this dwarf galaxy. If they were calibrated on LMC C stars, our objects would have distances and $Z$ values $\sim 25 \%$ larger. The scatter on the adopted absolute magnitude $M_{\mathrm{K}_{\mathrm{s}}}$ is around 0.3-0.4 $(1 \sigma)$. Concerning the $\mathrm{S}$ star (\# 80), it was assumed that its $M_{\mathrm{K}_{\mathrm{s}}}$ was similar to that of C stars.

For each star, interstellar colour excesses $E_{B-V}$ were taken from the maps of Schlegel et al. (1998) and a colour excess of 0.13 was adopted for LMC stars. The $J$ and $K_{\mathrm{s}}$ extinctions were 
Table 2. List of known very red C stars with $J-K_{\mathrm{s}}>3, f_{12}<7 \mathrm{Jy}$, and located out of the Galactic plane. After the names and galactic coordinates, this table provides the IRAS $12 \mu \mathrm{m}$ flux, $K_{\mathrm{s}}$ and $J-K_{\mathrm{s}}$ from 2MASS, distances $d$ and heights $Z$ above the Galactic plane.

\begin{tabular}{|c|c|c|c|c|c|c|c|c|c|}
\hline IRAS name & 2MASS name & $\begin{array}{c}l \\
\text { (deg) }\end{array}$ & $\begin{array}{c}b \\
\text { (deg) }\end{array}$ & $\begin{array}{r}f_{12} \\
(\mathrm{Jy})\end{array}$ & $\begin{array}{c}K_{\mathrm{s}} \\
(\mathrm{mag})\end{array}$ & $\begin{array}{r}J-K_{\mathrm{s}} \\
(\mathrm{mag})\end{array}$ & $\begin{array}{c}d \\
(\mathrm{kpc})\end{array}$ & $\begin{array}{c}Z \\
(\mathrm{kpc})\end{array}$ & $\begin{array}{l}\text { Note } \\
-\end{array}$ \\
\hline $01582+0931$ & 2MASS J020056.14+094535.6 & 149.845 & -49.446 & 2.27 & 7.17 & 3.06 & 7 & -5.5 & \#62 \\
\hline $03242+1429$ & 2MASS J032659.91+143956.9 & 169.816 & -33.690 & 0.59 & 8.21 & 3.41 & 13 & -7.5 & 1 \\
\hline $03582+1819$ & 2MASS J040109.72+182808.1 & 173.496 & -25.252 & 1.06 & 9.25 & 4.66 & 17 & -7.5 & 1 \\
\hline $04188+0122$ & 2MASS J042127.25+012913.4 & 192.177 & -31.987 & 3.37 & 6.42 & 3.28 & 6 & -3.5 & 2 \\
\hline $08427+0338$ & 2MASS J084522.27+032711.2 & 223.486 & +26.816 & 6.50 & 6.25 & 3.41 & 6 & +2.5 & 3 \\
\hline $08546+1732$ & 2MASS J085725.82+172051.9 & 210.261 & +35.437 & 0.57 & 10.71 & 4.41 & 36 & +21.0 & 4 \\
\hline $12560+1656$ & 2MASS J125833.50+164012.2 & 312.253 & +79.413 & 0.77 & 7.82 & 3.48 & 11 & +11.0 & 5 \\
\hline not IRAS & 2MASS J194850.65-305831.7 & 9.433 & -25.076 & $<0.3$ & 10.21 & 3.39 & 25 & -10.0 & 6 \\
\hline $20005+7737$ & 2MASS J195840.16+774526.2 & 110.190 & +23.019 & 0.50 & 8.69 & 3.35 & 14 & +5.5 & \#77 \\
\hline 20176-1458 & 2MASS J202027.66-144927.2 & 029.047 & -26.265 & 0.45 & 8.71 & 3.14 & 14 & -6.0 & 6 \\
\hline 21064+7749 & 2MASS J210522.23+780116.2 & 112.308 & +20.130 & 5.88 & 6.12 & 3.66 & 4 & +1.3 & \#79 \\
\hline not IRAS & 2MASS J215526.97+234214.4 & 78.911 & -23.760 & & 5.50 & 3.40 & 3 & -1.3 & \#82 \\
\hline
\end{tabular}

Notes: (1) discovered by Liebert et al. (2000); (2) discovered by TI98 as APM 0418+0122; (3) this star is CGCS 6306 in the Catalogue of Galactic carbon stars, Alksnis et al. (2001); (4) discovered by Cutri et al. (1989); (5) discovered by Beichman et al. (1990); (6) discovered by Mauron et al. (2004).

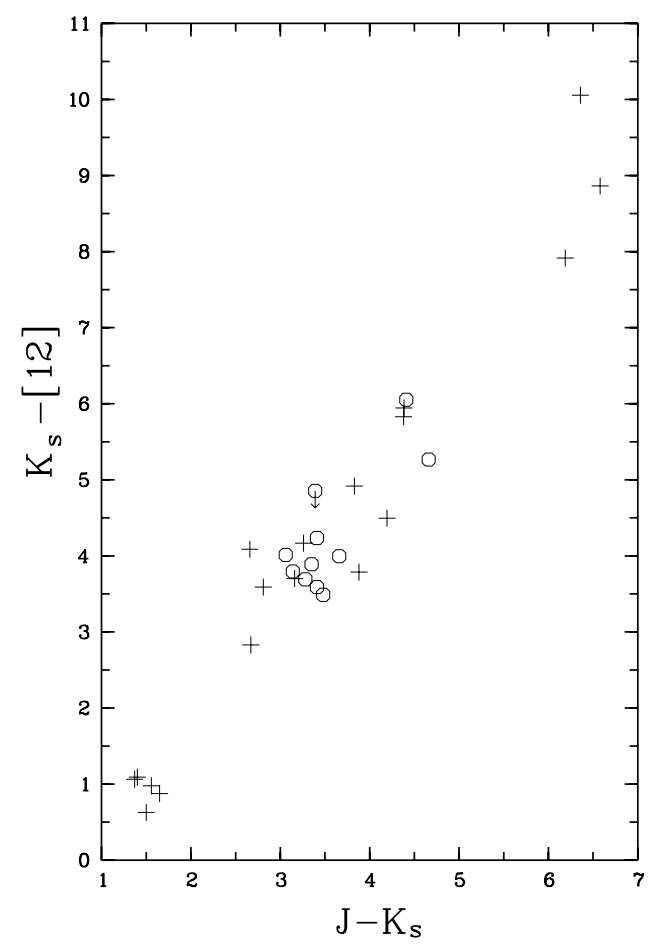

Fig. 4. Colour colour diagram $K_{\mathrm{s}}-[12]$ versus $J-K_{\mathrm{s}}$. Crosses represent a subsample of bright carbon stars of Groenewegen et al. (1992). Circles indicate the objects of Table 2, which appear to follow the trend of the bright carbon stars

calculated as $A_{J}=0.902 E_{B-V}$ and $A_{K_{\mathrm{s}}}=0.367 E_{B-V}$. In general, these corrections for interstellar reddening are low, but for a few stars $E_{B-V}$ is of the order of $0.5-0.6 \mathrm{mag}$, so that $A_{K_{\mathrm{s}}}$ reaches $0.2 \mathrm{mag}$ and cannot be neglected. Table 3 shows the adopted values of $E_{B-V}$, the dereddened colour $\left(J-K_{\mathrm{s}}\right)_{0}$, the absolute magnitude $M_{K_{\mathrm{s}}}$ and the derived distance $d$ in kpc. Due to the scatter on $M_{K_{\mathrm{s}}}$, the uncertainty on distances is about 20 percent $(1 \sigma)$.

It can be seen in Table 3 that distances are from $\sim 5 \mathrm{kpc}$ to $\sim 110 \mathrm{kpc}$. Two stars have $d<5 \mathrm{kpc}, 15$ have $d$ between 5 and $20 \mathrm{kpc}$ and 8 have $d$ between 20 and $110 \mathrm{kpc}$. Some of these distances could be improved in the future if the stars are shown to be periodic variables. For example, if we assume that stars \#60 and \#78 (with light curves in Fig. 3) are periodic, we can apply the $K$-band period-luminosity relation for semiregular variables established by Knapp et al. (2003). The NSVS light curves suggest that their periods could be 240 and 320 days, respectively. Then, with the $P-M_{K}$ relation $M_{K}=-1.34 \log P-4.5$, and assuming that the (average) $K$ magnitude is not too different from $K_{\mathrm{s}}$, we find for $\# 60, M_{K}=-7.69, d=7.6 \mathrm{kpc}$, a distance close to the one in Table $3(5.9 \mathrm{kpc})$. As for \#78, we obtain $M_{K}=-7.86$, $d=14.7 \mathrm{kpc}$, and this distance is $40 \%$ larger than that found in Table $2(10.5 \mathrm{kpc})$.

Galactocentric $X Y Z$ coordinates were calculated in the system of Newberg et al. (2003), in which the Sun is at $X=-8.5 \mathrm{kpc}, Y=0, Z=0$; the $Y$-axis is positive towards $l=+90^{\circ}$ and the $Z$ axis is positive to $b=+90^{\circ}$. Figure 5 displays the $Y Z$ and $X Z$ plots of previously known $\mathrm{C}$ stars and those of Table 1. In this system, the Sgr Stream as traced with the M-type giants (Majewski et al. 2003) is approximatively given by the following equation (Newberg et al. 2003):

$-0.064 X+0.970 Y+0.233 Z+0.232=0$.

In Table 3, last column, the distance $D$ of each object to this average plane is derived. We chose $D$ to have the sign of $Y$. It can be seen that $D$ is relatively small when compared with the range of distances. More specifically, out of 25 stars, there are 14 with $D<5 \mathrm{kpc}$. Some stars are quite distant from the Sun, like \#65 at $53 \mathrm{kpc}$ and \#69 at $51 \mathrm{kpc}$, but their $D$ is very small, 4 and $-2 \mathrm{kpc}$. This suggests that they belong to the Sgr Stream, although supplementary observations, especially radial velocities, are needed to confirm this.

There are two new stars that are especially distant, \#59 at $\sim 110 \mathrm{kpc}$ and $\# 81$ at $\sim 95 \mathrm{kpc}$. Figure 5 shows that four carbon stars are now known in the region $Z<-60 \mathrm{kpc}$ and none are known in the symetrical region $Z>+60 \mathrm{kpc}$, although a large number have been discovered closer to us and with $0 \lesssim Z \lesssim$ $50 \mathrm{kpc}$. This asymmetry qualitatively supports the existence of an extended branch of the Sgr Stream as in the model of Law et al. (2005, see Fig. 4 of Mauron et al. 2005). The width of this branch along the $X$ direction is $\sim 90 \mathrm{kpc}$, which is in fair agreement with the positions of our four $\mathrm{C}$ stars. 
Table 3. Properties of the halo $\mathrm{C}$ stars. The table lists the running number, the galactic coordinates $l$ and $b$ in degrees, the interstellar colour excess measured in the direction of the object, $E_{B-V}$ from Schlegel et al. maps, the dereddened $J-K_{\mathrm{S}}$ colour, the adopted $K_{\mathrm{s}}$-band absolute magnitude, the distance $d$ to the Sun in kpc, the galactocentric $X Y Z$ coordinates in kpc, and the distance $D$ to the Sgr Stream average plane, in kpc.

\begin{tabular}{rcccccrrrrr}
\hline \hline No. & $l$ & $b$ & $E_{B-V}$ & $\left(J-K_{\mathrm{s}}\right)_{0}$ & $M_{K_{\mathrm{s}}}$ & \multicolumn{1}{c}{$d$} & $X$ & $Y$ & $Z$ & $D$ \\
\hline 59 & 114.351 & -61.453 & 0.020 & 1.329 & -6.97 & 111.8 & -30.5 & 48.7 & -98.2 & 26.5 \\
60 & 127.393 & -22.689 & 0.050 & 1.429 & -7.15 & 5.9 & -11.8 & 4.3 & -2.3 & 4.7 \\
61 & 135.175 & -24.325 & 0.053 & 1.611 & -7.42 & 18.0 & -20.2 & 11.6 & -7.4 & 11.0 \\
62 & 149.845 & -49.446 & 0.068 & 3.060 & -7.16 & 7.3 & -12.6 & 2.4 & -5.5 & 2.1 \\
63 & 163.123 & -58.546 & 0.032 & 1.478 & -7.25 & 28.1 & -22.5 & 4.3 & -24.0 & 0.2 \\
64 & 162.049 & -36.082 & 0.291 & 1.183 & -6.56 & 22.4 & -25.7 & 5.6 & -13.2 & 4.2 \\
65 & 172.347 & -28.475 & 0.316 & 1.832 & -7.64 & 53.1 & -54.8 & 6.2 & -25.3 & 3.9 \\
66 & 165.713 & -21.582 & 0.164 & 1.349 & -7.01 & 14.1 & -21.2 & 3.2 & -5.2 & 3.5 \\
67 & 181.911 & -31.637 & 0.289 & 1.415 & -7.13 & 11.1 & -17.9 & -0.3 & -5.8 & -0.3 \\
68 & 176.133 & -25.619 & 0.472 & 1.284 & -6.89 & 8.9 & -16.5 & 0.5 & -3.9 & 0.9 \\
69 & 181.245 & -23.296 & 0.587 & 1.697 & -7.53 & 50.6 & -55.0 & -1.0 & -20.0 & -1.9 \\
70 & 190.204 & -20.452 & 0.164 & 1.502 & -7.28 & 14.6 & -21.9 & -2.4 & -5.1 & -1.9 \\
71 & 140.861 & +22.738 & 0.177 & 1.417 & -7.13 & 9.8 & -15.5 & 5.7 & 3.8 & 7.7 \\
72 & 161.381 & +21.086 & 0.080 & 1.363 & -7.03 & 10.3 & -17.7 & 3.1 & 3.7 & 5.2 \\
73 & 169.337 & +22.917 & 0.071 & 1.545 & -7.33 & 8.5 & -16.2 & 1.5 & 3.3 & 3.5 \\
74 & 26.142 & +22.867 & 0.316 & 1.188 & -6.58 & 21.5 & 9.3 & 8.7 & 8.4 & 10.1 \\
75 & 48.195 & +21.356 & 0.082 & 1.399 & -7.10 & 10.7 & -1.9 & 7.4 & 3.9 & 8.4 \\
76 & 107.672 & +23.332 & 0.093 & 1.593 & -7.39 & 10.7 & -11.5 & 9.3 & 4.2 & 11.0 \\
77 & 110.190 & +23.019 & 0.155 & 3.273 & -7.08 & 13.9 & -12.9 & 12.0 & 5.4 & 13.9 \\
78 & 109.469 & +21.659 & 0.218 & 1.458 & -7.21 & 10.5 & -11.8 & 9.2 & 3.9 & 10.8 \\
79 & 112.308 & +20.130 & 0.548 & 3.370 & -7.03 & 3.9 & -9.9 & 3.4 & 1.3 & 4.5 \\
80 & 68.200 & -21.673 & 0.092 & 2.258 & -7.50 & 5.8 & -6.5 & 5.0 & -2.2 & 5.0 \\
81 & 37.019 & -39.965 & 0.069 & 1.382 & -7.07 & 95.6 & 50.0 & 44.1 & -61.4 & 25.5 \\
82 & 78.911 & -23.760 & 0.066 & 3.363 & -7.04 & 3.2 & -7.9 & 2.9 & -1.3 & 3.2 \\
83 & 83.298 & -28.940 & 0.058 & 2.130 & -7.56 & 25.8 & -5.9 & 22.4 & -12.5 & 19.5 \\
\hline
\end{tabular}
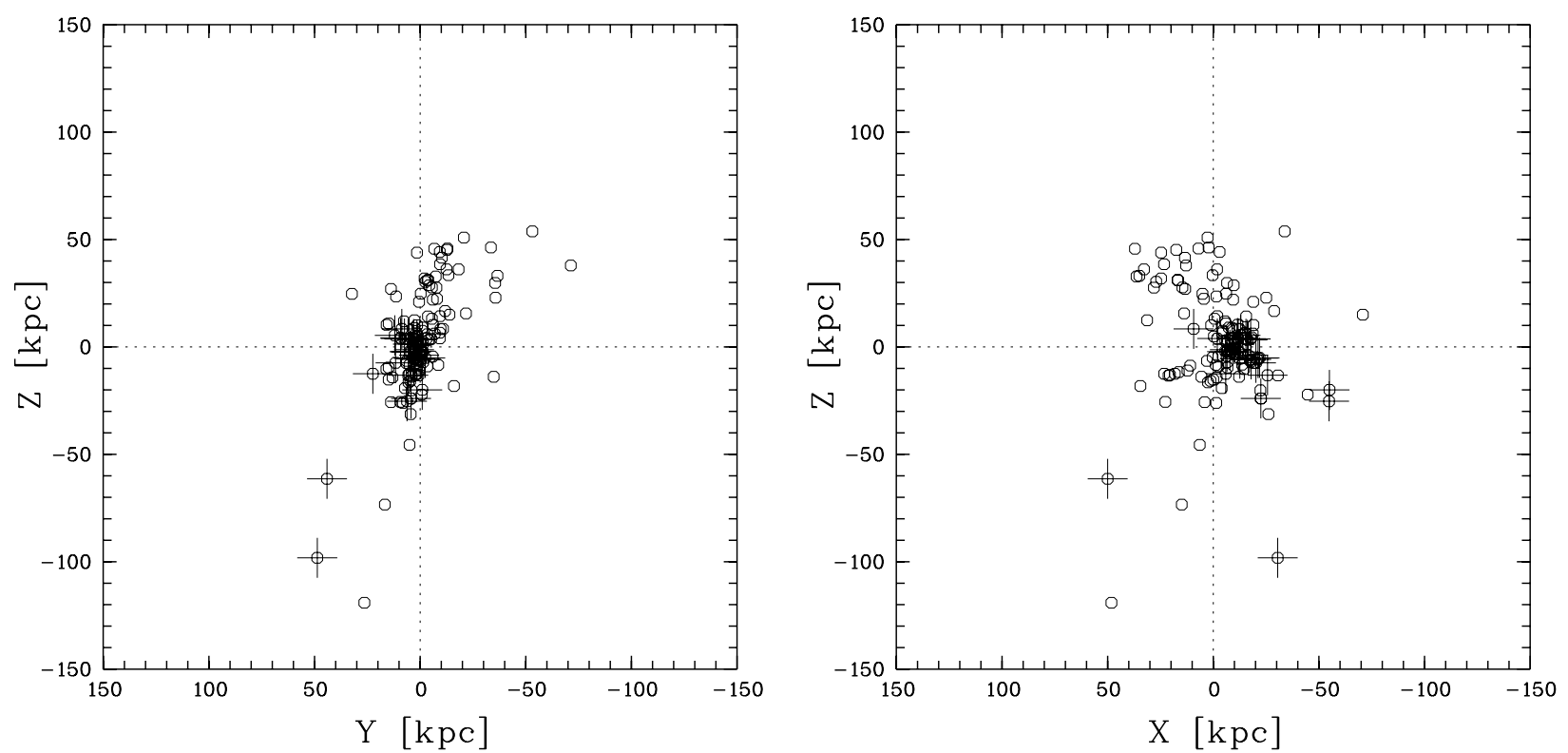

Fig. 5. Plots in galactocentric $X Y Z$ of the observed halo C stars, with those found in this paper indicated by circle with a overplotted cross. Left panel: in this $Z$ versus $Y$ the Sgr Stream is seen nearly edge-on, slightly tilted clockwise with respect to the $Z$ axis. Note that $Y$ is positive to the left. The two new very distant C stars found in this paper(\# 59 and \#81) are on the lower left. Their distances to the main plane of the Sgr Stream are about $25 \mathrm{kpc}$. Right panel: $Z$ versus $X$ plot. $X$ is positive to the left and $X_{\odot}=-8.5 \mathrm{kpc}$. The positions of the $\mathrm{C}$ stars at $Z<-50 \mathrm{kpc}$ suggest that the $\mathrm{Sgr}$ debris have a broad extent, $\sim 100 \mathrm{kpc}$, along the $\mathrm{X}$-coordinates.

We note however that, recently, the Sgr Stream has been mapped by analysing the data of Sloan survey (Belokurov et al. 2006; Fellhauer et al. 2006). The map puts strong constraints on the Stream geometry and is well explained within a model where there is no matter at $Z<-60 \mathrm{kpc}$. It is then possible that our four
C stars with high negative $Z$ are part of another stream. It will be interesting in the future to see if any new distant star can be detected close to them. In both cases, the fact that cool carbon stars are found indicate that an intermediate-age population is present in this region of the halo. 


\section{Conclusions}

By searching the 2MASS catalogue for rare cool carbon stars located out of the Galactic plane $\left(|b|>20^{\circ}\right)$, we have found 23 new cases with $K_{\mathrm{s}}$ magnitudes covering the range $\sim 6$ to 13.3 . Their colours and spectra are typical of AGB stars, which is also supported by important variability for those objects bright enough to be detected by the NSVS survey.

We have discovered four cases of $\mathrm{C}$ stars with $J-K_{\mathrm{s}}>3$ and located well above the Galactic plane. After including those already published in the literature, we could made a list of $12 \mathrm{ob}-$ jects with similar properties. Previous works (Groenewegen et al. 1997) show that two of them are deficient in oxygen and one has a very small wind expansion velocity. This suggests that some (or all) members of this sample could be metal deficient as well. Because they are closer to us than the Magellanic Clouds, supplementary observations of these stars, especially their $\mathrm{CO}$ emission, could help to better know AGB winds at low metallicity.

Distances were estimated by using the 2MASS $J$ and $K_{\mathrm{s}}$ data and by assuming our objects to be similar to those of the Sagittarius dwarf galaxy. It is found that two objects are at remarkably large distances from the Sun $(\sim 100 \mathrm{kpc})$. Their galactocentric $X Y Z$ coordinates suggest that they might belong, together with two other distant carbon stars previously found, to a distant loop of the Sgr stream extending at large negative $Z$. Additional detections of distant stars in this region of the halo should help to confirm this conclusion.

Acknowledgements. We would like to thank our referee, E. Lagadec, for useful comments. We thank also the staff of Observatoire de Haute-Provence, which is supported by the French Centre National de Recherche Scientifique. We acknowledge the use of the Two Micron All Sky Survey (2MASS) which is a joint project of the University of Massachusetts and the Infrared Processing and Analysis Centre/California Institute of Technology, funded by the National Aeronautics and Space Administration (NASA) and the National Science Foundation (NSF). This publication also makes use of the data from the Northern Sky Variability Survey (NSVS) created jointly by the Los Alamos National Laboratory and University of Michigan. The NSVS was funded by the Department of Energy, NASA and NSF. Finally, this work benefited from using the CDS database of Strasbourg (France).

\section{References}

Alksnis, A., Balklavs, A., Dzervitis, U., et al. 2001, Baltic Aston., 10, 1 (CDS catalogue III/227)

Beichman, C.A., Chester, T., Gillet, F.C., et al. 1990, AJ, 99, 1569

Belokurov, V., Zucker, D. B., Evans, N. W., et al. 2006, ApJ, 642, L137

Bergeat, J., Knapik, A., \& Rutily, B. 2002, A\&A, 385,94

Claussen, M. J., Kleinmann, S. G., Joyce, R. R., \& Jura, M. 1987, ApJS, 65,385

Cruz, K. L., Reid, I. N., Liebert, J., Kirkpatrick, J.D., \& Lowrance, P.J. 2003, AJ, 126,2421

Cutri, R. M., Low, F. J., Kleinmann, S. G., et al. 1989, AJ, 97, 866

Cutri, R. M., Skrutskie, M. F., Van Dyk, S., et al. 2003, University of Massachusetts and Infrared Processing and Analysis Center (IPAC, California Institute of Technology) (CDS catalogue II/246)

Fellhauer, M., Belokurov, V., Evans, N. W., et al. 2006, ApJ, 651, 167

Groenewegen, M. A. T. 2005, Populations of AGB stars and LPVs in the Galaxy and the Local Group [arXiv:astro-ph/0506381]

Groenewegen, M. A. T., de Jong, T., van der Bliek, N. S., Slijkhuis, S., \& Willems, F. J. 1992, A\&A, 253, 150

Groenewegen, M. A. T., Oudmaijer, R. D., \& Ludwig, H. G. 1997, MNRAS, 292, 696

Groenewegen, M. A. T., Sevenster, M., Spoon, H. W. W., \& Pérez, I. 2002, A\&A, 390,511

Habing, H. J. 1996, A\&ARv, 7, 97

Ibata, R., Lewis, G. F., Irwin, M., Totten, E., \& Quinn, T. 2001, ApJ, 551, 294

Irwin, M. J., 2000, The APM Catalogue [http://www.ast.cam.ac.uk/ apmcat/]

Knapp, G. R., Pourbaix, D., Platais, I., \& Jorissen, A. 2003, A\&A, 403, 993

Law, D. R., Johnston, K. V., \& Majewski, S. R. 2005, ApJ, 619, 807

Liebert, J., Cutri, R. M., Nelson, B., et al. 2000, PASP, 112, 1315

Majewski, S. R., Skrustkie, M. F., Weinberg, M. D., \& Ostheimer, J. C. 2003, ApJ, 599, 1082

Mauron, N., Azzopardi, M., Gigoyan, K., \& Kendall, T. R. 2004, A\&A, 418, 77

Mauron, N., Kendall, T. R., \& Gigoyan, K. 2005, A\&A, 438, 867

Monet, D., Bird, A., Canzian, B., et al. 1998, The USNO-A2.0 Catalogue, US Naval Observatory Flagstaff Station and Universities Space Research Association (CDS catalogue I/252)

Monet, D.G., Levine, S.E., Canzian, B., et al. 2003, AJ, 125, 984

Mouhcine, M., \& Lançon, A. 2003, MNRAS, 338, 572

Newberg, H.J., Yanny, B., Grebel, E. K., et al. 2003, ApJ, 596, L191

Nikolaev, S., \& Weinberg, M. D. 2000, ApJ, 542, 804

Schlegel, D. J., Finkbeiner, D. P., \& Davis M. 1998, ApJ, 500, 525

Totten, E. J., \& Irwin M. J. 1998, MNRAS, 294, 1 (TI98)

Van den Bergh, S. 2000, The Galaxies of the Local Group (Cambridge: University Press)

Wallerstein, G., \& Knapp, G. R. 1998, ARA\&A, 36, 369

Woźniak, P. R., Vestrand, W. T., Akerlof, C. W., et al. 2004, AJ, 127, 2436 
N. Mauron et al.: Cool carbon stars in the halo, Online Material p 1

\section{Online Material}


Appendix A
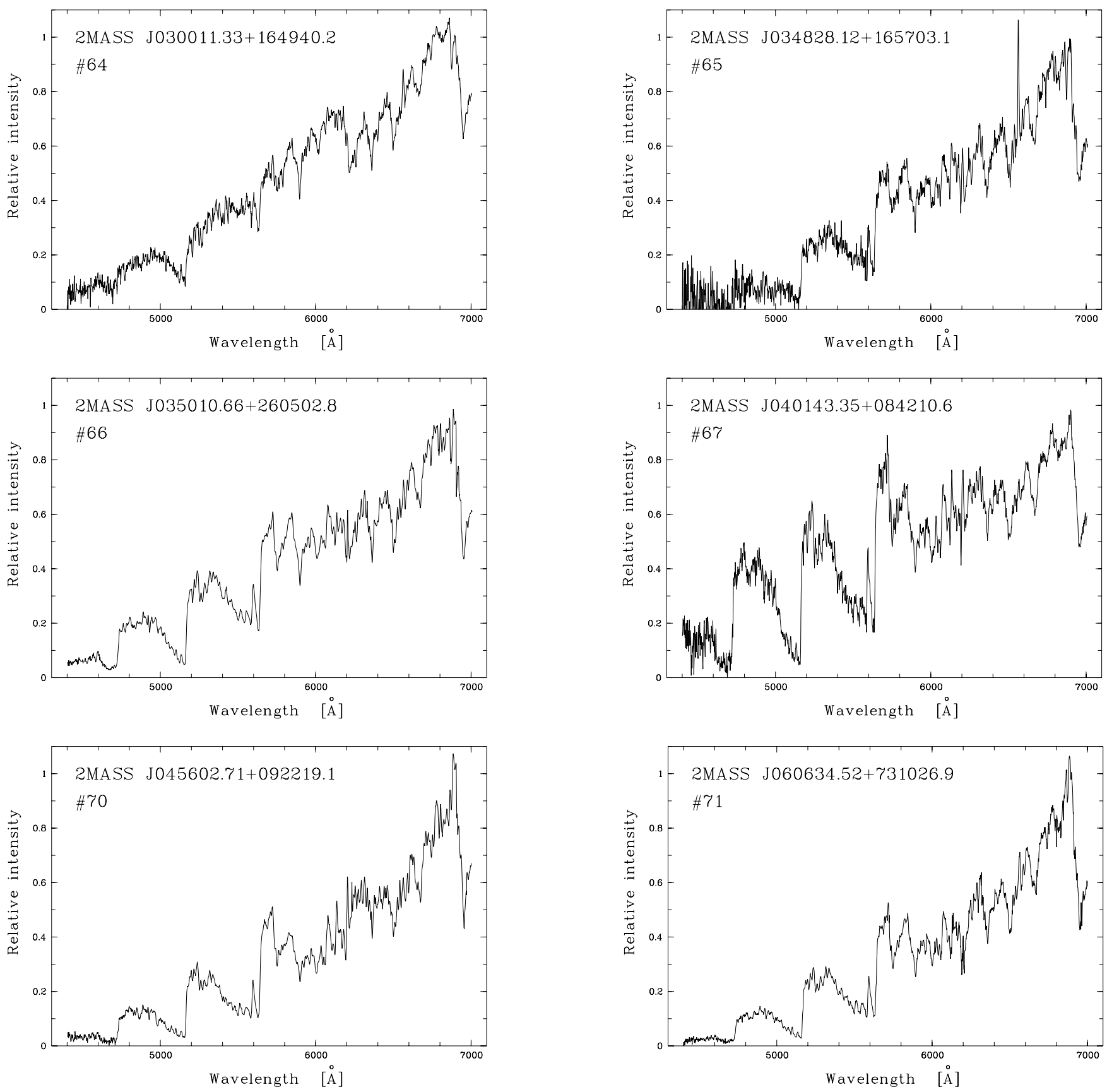

Fig. A.1. Stars observed at Byurakan. 
N. Mauron et al.: Cool carbon stars in the halo, Online Material p 3
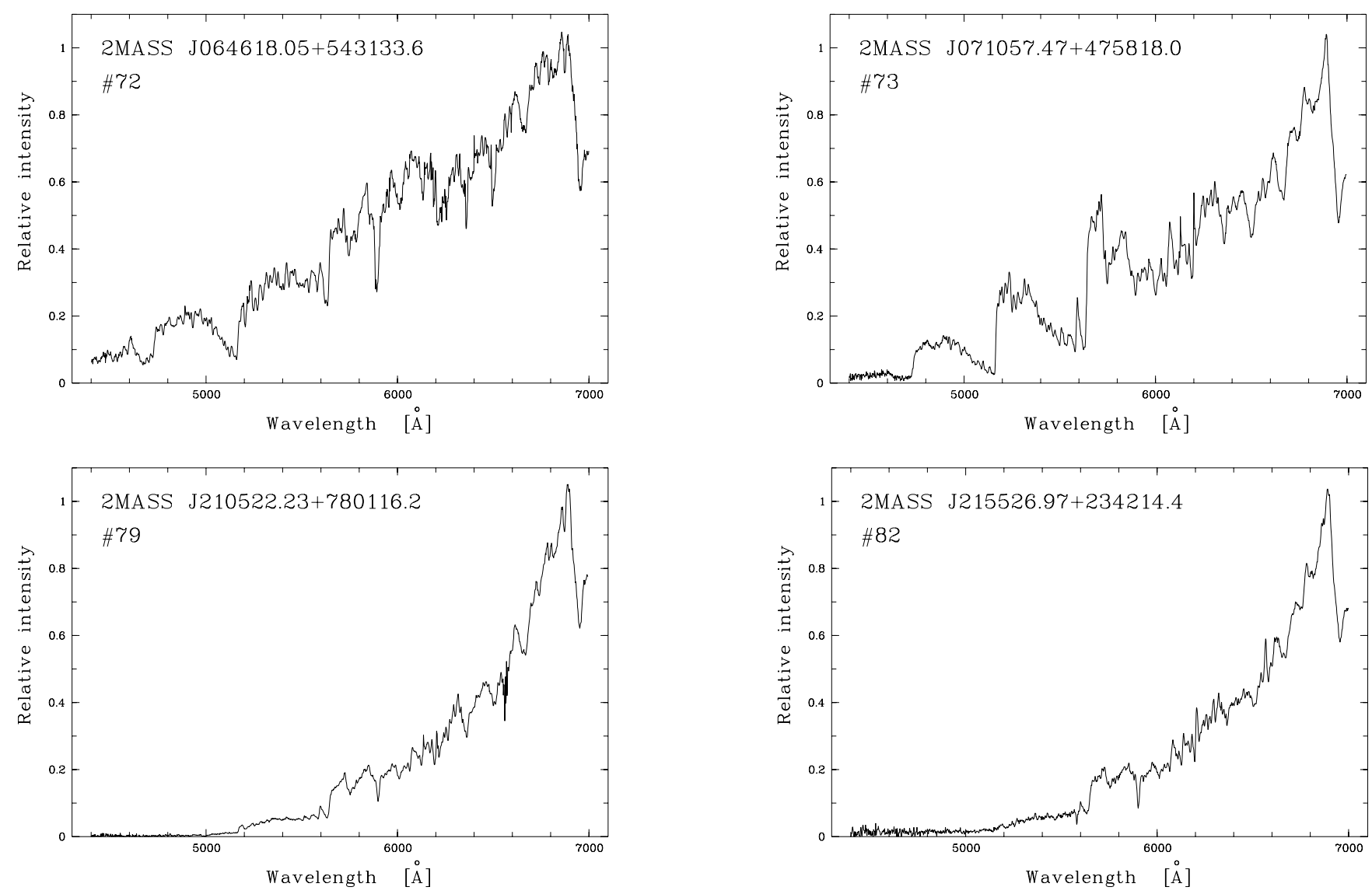

Fig. A.1. continued. 
N. Mauron et al.: Cool carbon stars in the halo, Online Material p 4
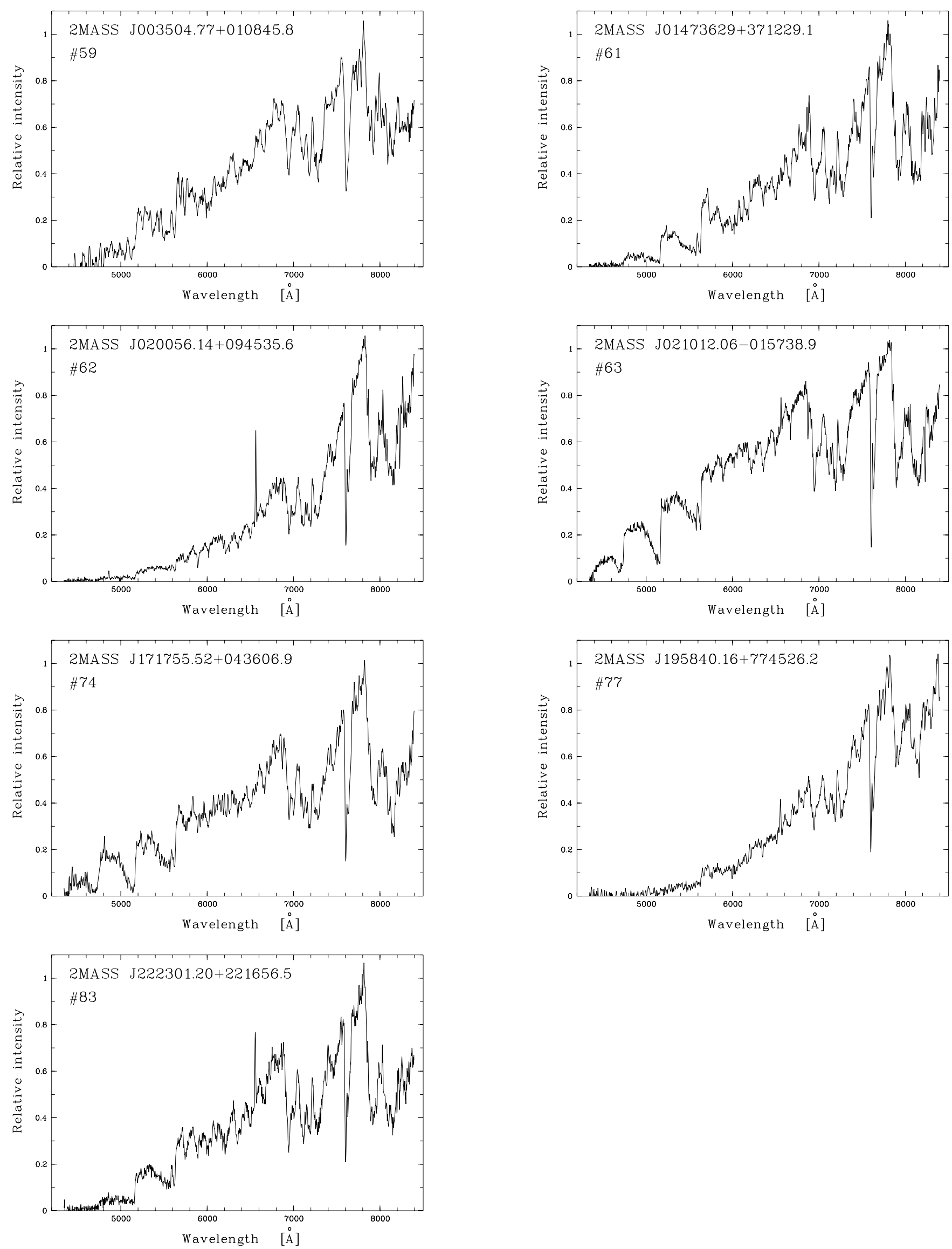

Fig. A.2. Stars observed at OHP in September 2005. 
N. Mauron et al.: Cool carbon stars in the halo, Online Material p 5
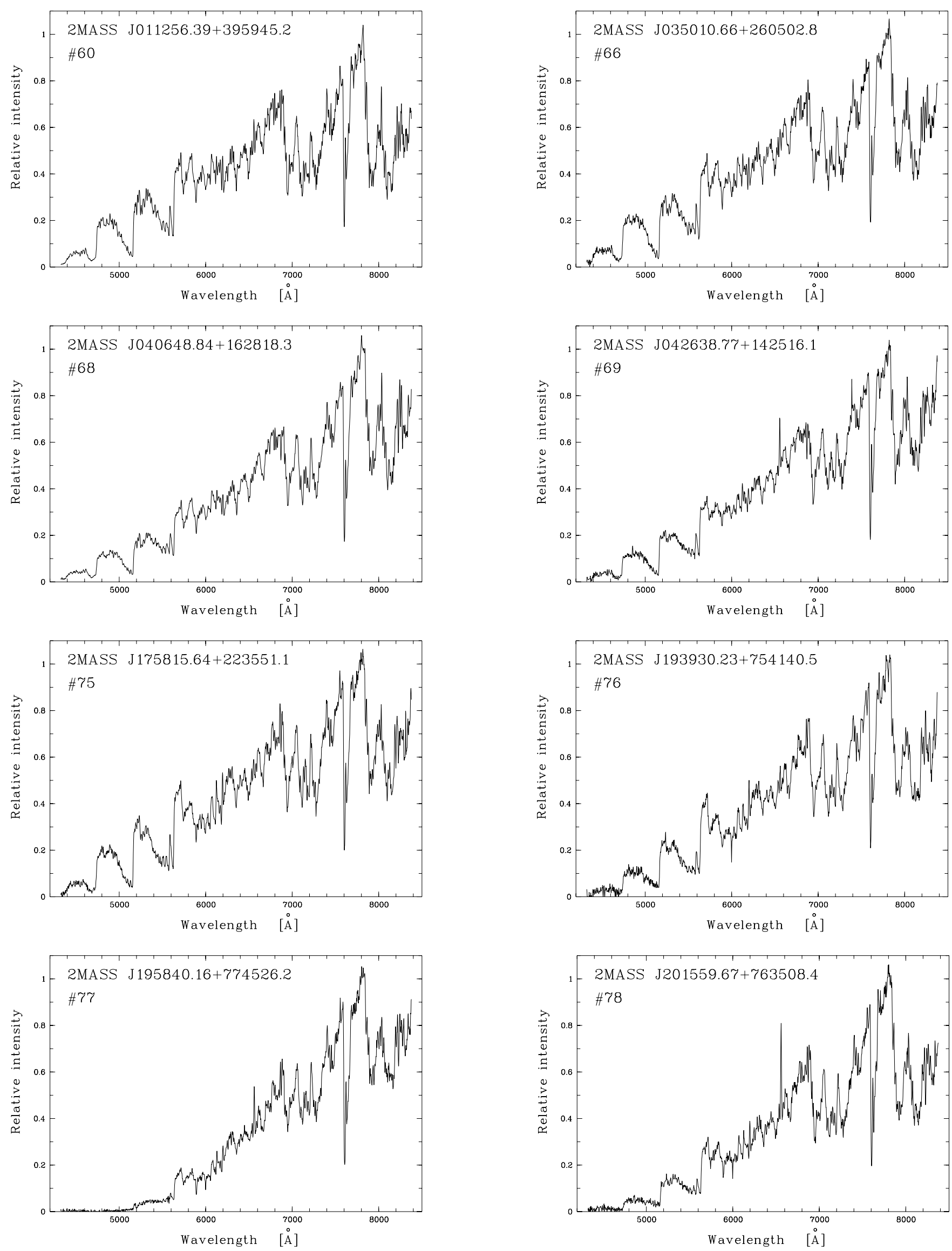

Fig. A.3. Stars observed at OHP in September 2006. 
N. Mauron et al.: Cool carbon stars in the halo, Online Material p 6
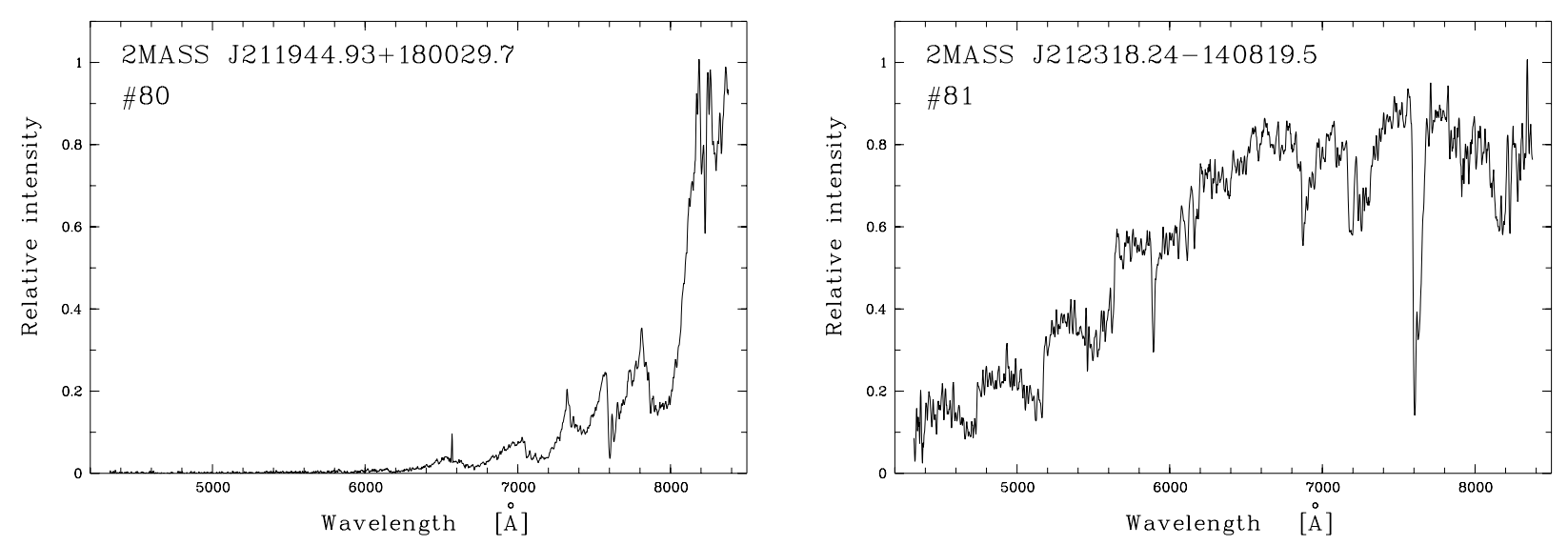

Fig. A.3. continued 
N. Mauron et al.: Cool carbon stars in the halo, Online Material $p 7$
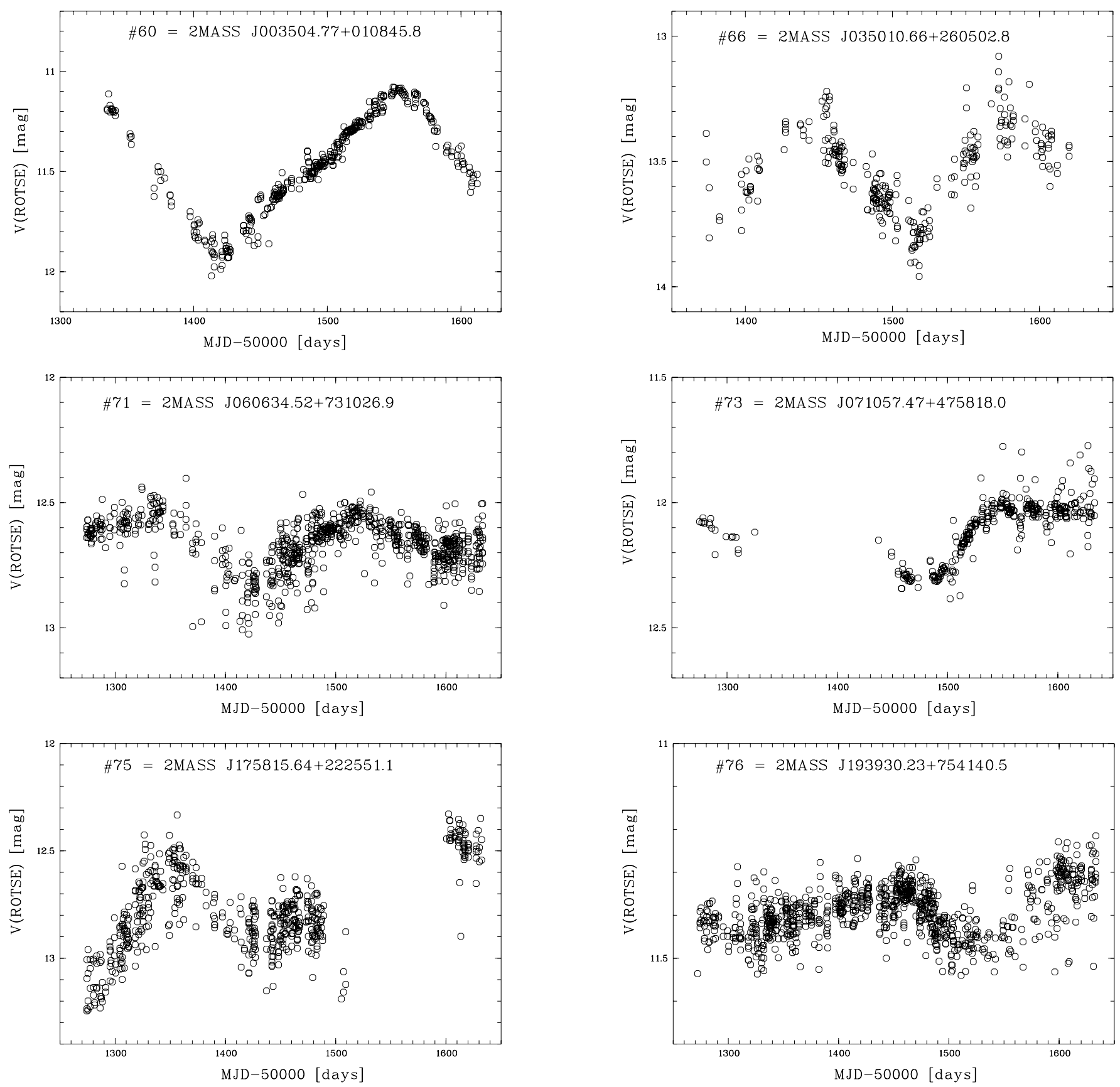

Fig. A.4. Light curves from NSVS data. 
N. Mauron et al.: Cool carbon stars in the halo, Online Material $p 8$
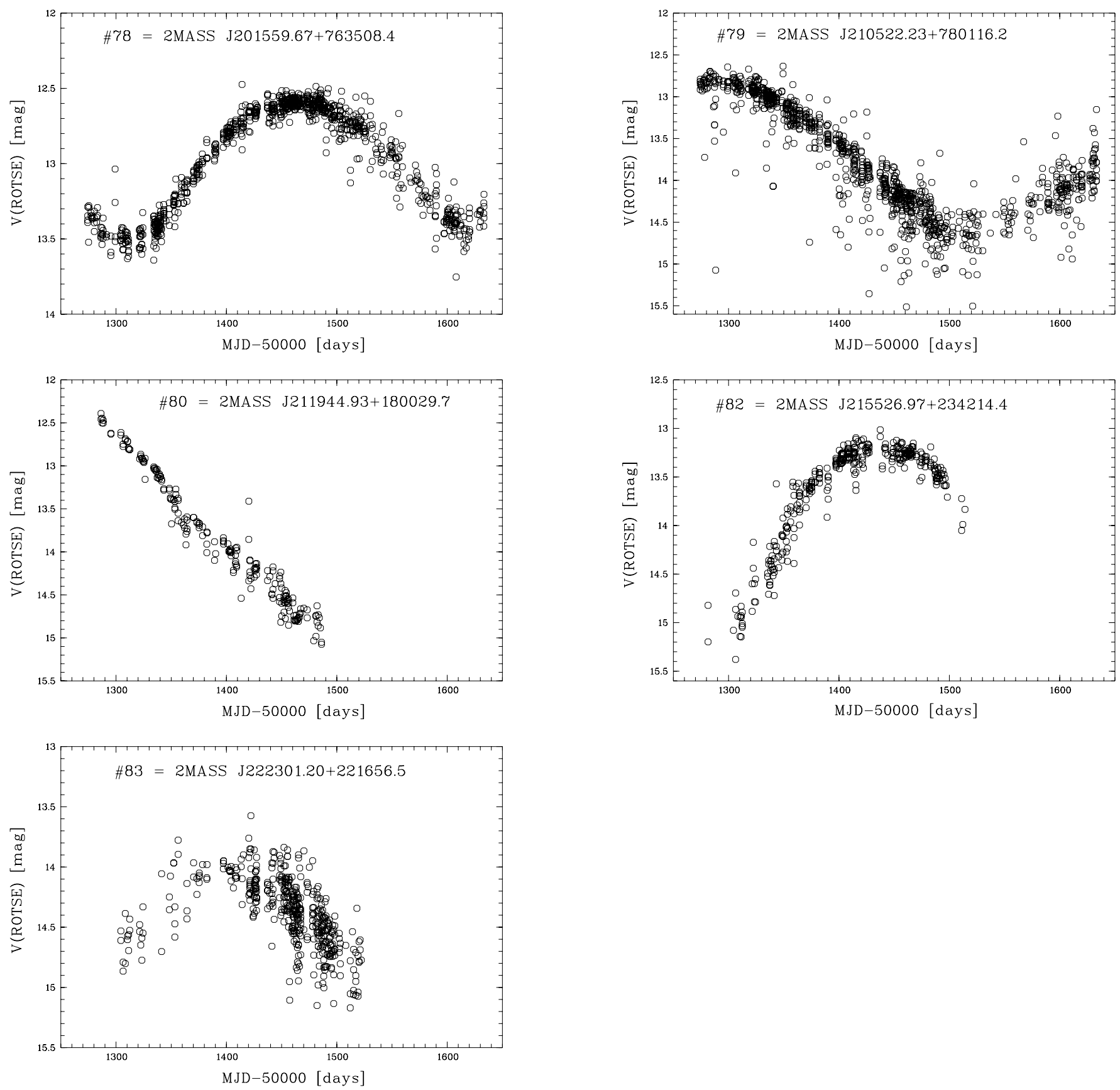

Fig. A.4. continued. 